\title{
Performance Comparison of Single \& Hybrid Optical Amplifiers for DWDM System Using Optisystem
}

\author{
Anil Agarwal $^{1}$, Sudhir Kumar Sharma ${ }^{2}$ \\ ${ }^{I}$ (Assistant Professor, Dept. of Electronics \& Comm. Engineering, Jaipur National University, Jaipur, India) \\ ${ }_{2}^{2}$ (Prof. (Dr.), Head, Dept. of Electronics \& Communication Engineering, Jaipur National University, Jaipur, \\ India)
}

\begin{abstract}
In this paper, single and hybrid amplifier configurations are implemented with FRA and EDFA amplifiers for 160 channel DWDM systems at $10 \mathrm{Gbps} \& 25 \mathrm{GHz}$ frequency spacing for L-band. These configurations are transmitted and measured over different distances for 50, 100, 150, 200 and $250 \mathrm{~km}$. The performance of single and hybrid optical amplifiers evaluated in terms of output power, BER, eye height and $Q$ factor. Among these setups EDFA-EDFA performed better than other optical amplifiers at $150 \mathrm{~km}$ distance. It provides output power (36.55 to -3.45 dBm), least BER (-38.96 to 0), large $Q$ factor (12.71 to 0$)$ and good eye diagram for different transmission distance ranging from 50 to $250 \mathrm{~km}$. These are all setups are arranged without using any gain-flattening technique and receiver filters.
\end{abstract}

Keywords-FRA, EDFA, DWDM, Transmission Distance, Output Power, BER, Eye Height, Q factor.

\section{Introduction}

There has been a strong demand in high capacity signal transmission systems and networks in recent. To increase data transmission capacities, several methods are proposed by adding more channels in the wavelength division multiplexing (WDM) system, so that spectral efficiency needs to be upgraded. To overcome these problems, the DWDM systems have been demonstrated using several types of wideband optical amplifiers [1]. The high speed transmission over the global telecommunication network without repeaters will continue to grow at an exponential rate and only optical fiber amplifiers will be able to meet the challenge [2]. For long-haul optical transmission systems broadband hybrid amplifiers with multi-pump Raman amplification and EDFA have been a demanding technique. There are two main reasons of using Raman amplifier first is the ability to provide gain at broad wavelength and second is to broaden the amplification bandwidth by adding more pump wavelength [3]. Laser diode pumped erbium fiber amplifiers (EDFAs) will play a key role as power boosters, optical repeaters and preamplifiers in long distance optical fiber communications systems [4]. So, this work will target to performance comparison of single and hybrid optical amplifiers for higher transmission distance with improved power and BER for DWDM communication systems. In literature review, various techniques using number of different amplifiers with single or multiple pumping have been proposed to increase the transmission distance over the bandwidth region. Hari et al. [2] investigated the different optical amplifiers for less number of channels with large channel spacing using eye pattern, BER and Q factor and get optimized transmission distance to transmit the signal. Delavaux et al. [4] designed two HEDFA setups as power booster modules for repeater less data transmission for less bit rate. Lee et al. [5] compared the performance of three single pumps, Raman/EDFA hybrid amplifier recycling residual Raman pump in a cascaded EDF section located after and prior to DCF and Raman assisted EDFA with respect to gain, noise figure and BER. Martini et al. [3] presented different hybrid amplifier configurations with two and three pumps to receive a flat global gain for a large bandwidth region. Cheng et al. [6] demonstrated a bismuth-based erbium-doped fiber amplifier (BiEDFA) that operates in both the C- and L-band regions with an intermediate broadband fiber Bragg grating sensor to improve flat gain characteristics and to reduce the noise figure. Masuda et al. [7] proposed a wide band and gain flattened discrete hybrid optical amplifier for long-distance wavelength-division-multiplexed (WDM) optical communication systems. Simranjit singh et al. [8] presented a HOA model using two stages DRA-EDFA dense wavelength division multiplexing system to minimize the gain variation without using any gain flattening technique. Optical communication is an efficient medium for transmission in long haul applications, but there are some drawbacks regarding optical fibers and components which degrade the efficiency of the fiber optic communication system. So, all the above researchers use different setups with variable parameters to provide large transmission distance, high flat gain, less noise figure, less system cost and large gain bandwidth for multiple channels. But some of them used large channel spacing, less number of channels, costly components in their systems. To remove these problems we have investigated the various single and HOAs for DWDM system at reduced channel spacing and high bit rate with different transmission distances. In this paper we have investigated different models of single and hybrid amplifiers (RAMAN, EDFA) to achieve better output power, high $\mathrm{Q}$ factor, low BER with improved transmission distance without using any gain equalizers. After the 
introduction, simulation setup and parameters of Raman-EDFA is described in Section II. Section III covers the results of the experimental setup and conclusion gives in Section IV.

\section{Simulation Setup}

We used 160 channel transmitter and receiver model at 10 Gbps data rate with channel spacing of 25 GHz. A transmitter compound component is built up using sixteen transmitters and multiplex ten transmitter compounds for one hundred sixty transmitters. This transmitter compound component consists of the PRBS data source, NRZ electrical driver, CW laser source and external Mach-Zehnder modulator in each transmitter section. The electrical driver converts the logical input signal into an electrical signal. The $\mathrm{CW}$ laser sources generate the 160 laser beams at $187-190.975 \mathrm{THz}$ with $25 \mathrm{GHz}$ channel spacing [8].

The simulations setup of EDFA, RAMAN and Hybrid Optical amplifiers using compound component at different transmission distance are shown in Fig. 1.

160 Channel Transmitters at 1-mW input power
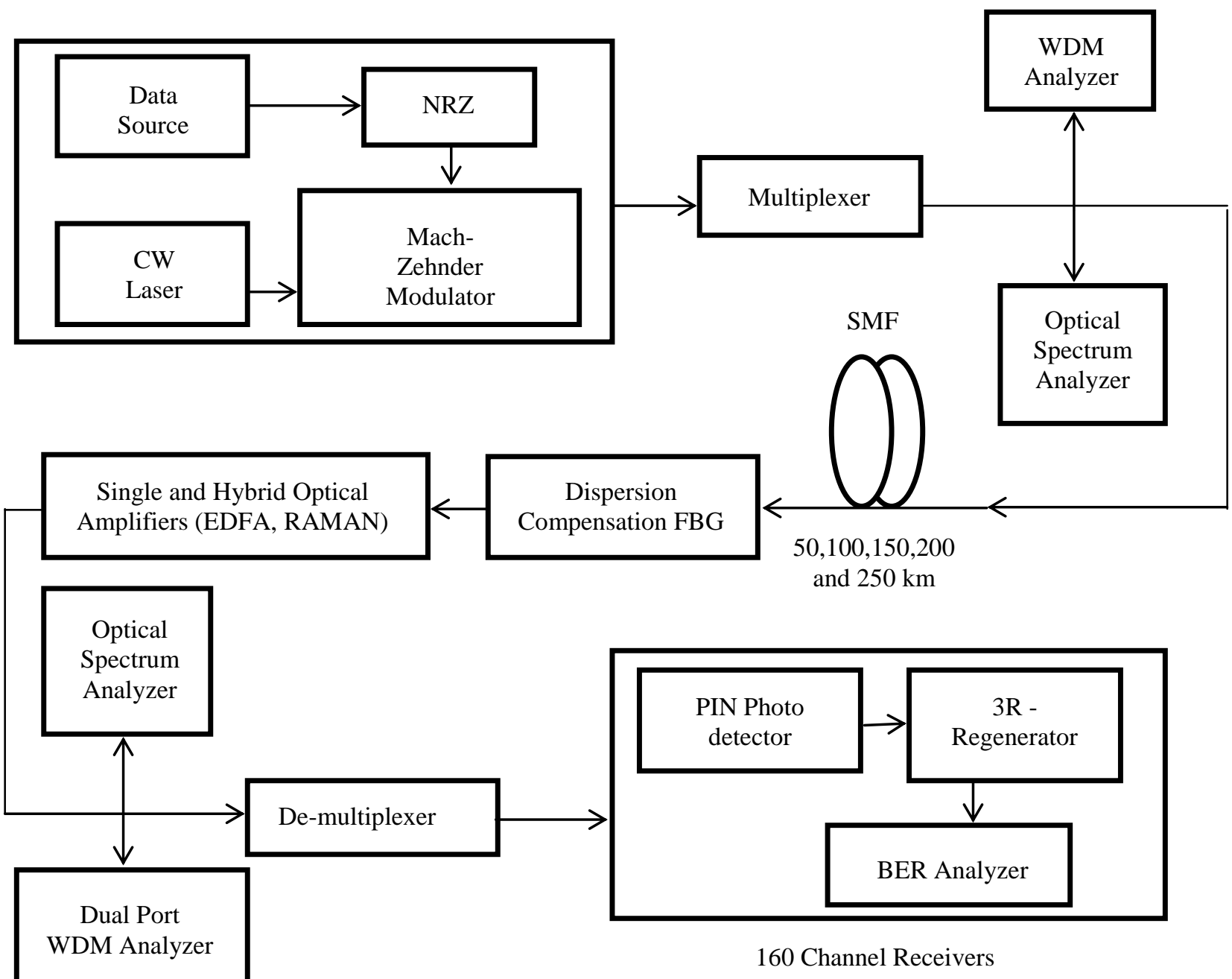

and $250 \mathrm{~km}$

Fig. 1. Block Diagram Showing Simulation Setup for Proposed Scheme

The output optical signal of the modulator is fed to the channel. This optical signal is transmitted and measured over different distance for 50,100,150, 200 and $250 \mathrm{~km}$ with nonlinearities individually. We used Dispersion fiber grating device to compensate dispersion. Then apply combination of EDFA and RAMAN as a Preamplifier to the channel. Dual port WDM analyzer, BER analyzer and Optical spectrum analyzer are used for measuring the signal power and spectrum at different levels. A compound receiver WDM Demux is used to detect all 160 signals and converts these into electrical form by using PIN photodiode. The setup is repeated for measuring the signal strength by using different amplifiers i.e. EDFA, RAMAN, EDFA-EDFA, EDFA- 
RAMAN, RAMAN-EDFA and RAMAN-RAMAN. Different results like gain, output power, eye height, Qfactor and BER show that EDFA-EDFA is the most suitable amplifier in the all proposed amplifiers.

Different components have different operational parameters. The SMF optical fiber is used to transmit the optical signal. Its various parameters are shown in Table I.

TABLE1

PARAMETERS FOR SMF OPTICAL FIBER

\begin{tabular}{|l|l|}
\hline Parameters & SMF \\
\hline Reference wavelength & $1550 \mathrm{~nm}$ \\
\hline SMF length & $100 \mathrm{~km}$ \\
\hline Attenuation & $0.2 \mathrm{~dB} / \mathrm{km}$ \\
\hline Dispersion & $16 \mathrm{ps} / \mathrm{nm} / \mathrm{km}$ \\
\hline PMD coefficient & $0.1 \mathrm{ps} / \mathrm{km}^{0.5}$ \\
\hline Nonlinear refractive index (n2) & $2.5 \mathrm{e}^{020} \mathrm{~m}^{2} / \mathrm{w}$ \\
\hline
\end{tabular}

The fixed gain EDFA is used to amplify the optical signal. Its various parameters are shown in Table II.

TABLE2

PARAMETERS FOR EDFA

\begin{tabular}{|l|l|}
\hline Operation mode & Gain control \\
\hline Gain shape & Flat \\
\hline Fixed small signal gain & $25 \mathrm{~dB}$ \\
\hline Noise figure & $4 \mathrm{~dB}$ \\
\hline
\end{tabular}

The Fiber RAMAN amplifier is also used to enhance the output power. Its various parameters are shown in Table III.

TABLE3

PARAMETERS OF RAMAN FIBER AMPLIFIER

\begin{tabular}{|l|l|}
\hline RAMAN fiber length & $22 \mathrm{~km}$ \\
\hline Attenuation & $0.2 \mathrm{~dB} / \mathrm{km}$ \\
\hline Temperature & $300 \mathrm{~K}$ \\
\hline Pump frequency & $207 \mathrm{THz}, 201 \mathrm{THz}$ \\
\hline Pump power & $650 \mathrm{~mW}, 250 \mathrm{~mW}$ \\
\hline
\end{tabular}

At the receiver side the PIN photo detector is used with a responsivity of $0.875 \mathrm{~A} / \mathrm{W}$ and dark current of $0.1 \mathrm{nA}$.

\section{Results}

The Performance of different single and hybrid amplifiers EDFA, RAMAN, EDFA-EDFA, RAMANEDFA, RAMAN-RAMAN and EDFA-RAMAN are evaluated and compared for $160 \times 10 \mathrm{Gbps}$ DWDM system in the term of received output power, Q Factor, eye height and minimum BER with nonlinearities at different transmission distance. The distance varied from 50 to $250 \mathrm{~km}$ in steps of $50 \mathrm{~km}$. To analyze the system, the results of the first channel have been taken.

The Fig.2 shows the graphical representation of output power as a function of length in the presence of nonlinearities. The output power is decreased due to the fiber nonlinearities and fiber attenuation. The better output power is provided by the EDFA-EDFA and RAMAN-EDFA amplifier for all over distance range. At 50 $\mathrm{km}$ output power by EDFA-EDFA and RAMAN-EDFA are $36.55 \mathrm{dBm}$ and $28.14 \mathrm{dBm}$ and also for the $250 \mathrm{Km}$ it becomes $-3.45 \mathrm{dBm}$ and $-9.14 \mathrm{dBm}$ respectively, But for other configurations power decreases linearly with length of the fiber. 


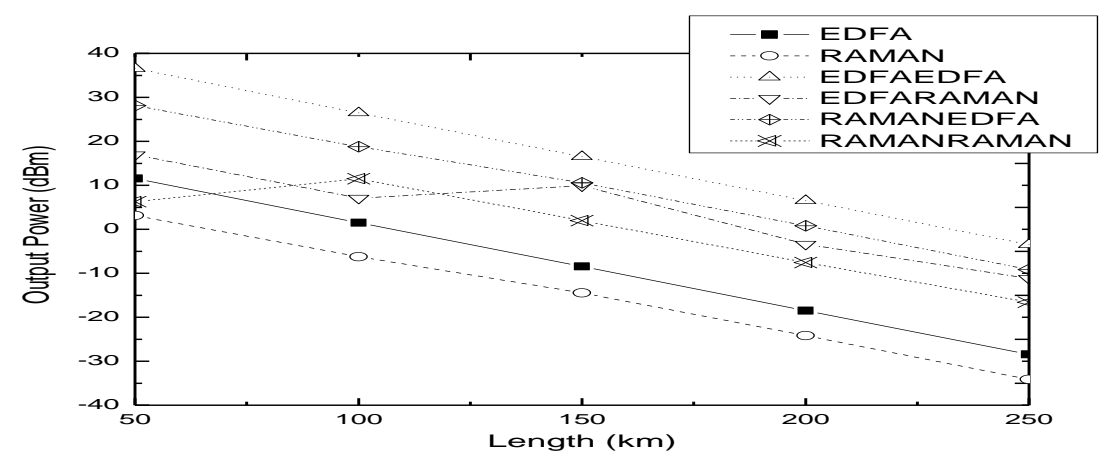

Fig. 2. Diagram Showing Output Power at Different Fiber Length

The variation in output power is 11.55 to -28.45 for EDFA, 3.14 to $-34.14 \mathrm{dBm}$ for RAMAN, 36.55 to -3.45 dBm for EDFA-EDFA, 16.92 to $-11.15 \mathrm{dBm}$ for EDFA-RAMAN, 28.14 to $-9.14 \mathrm{dBm}$ for RAMANEDFA, 6.29 to $-16.56 \mathrm{dBm}$ for RAMAN-RAMAN received by the proposed scheme.

The Fig. 3 shows the graphical representation of log of BER as a function of distance in the presence of nonlinearities. At $150 \mathrm{~km}$ the better BER is provided by the EDFA-RAMAN and EDFA-EDFA amplifier -25.34 and -20.90 respectively and for the $250 \mathrm{Km}$ it becomes zero. Other single and hybrid amplifiers also provides the acceptable BER but there is very much variation in BER.

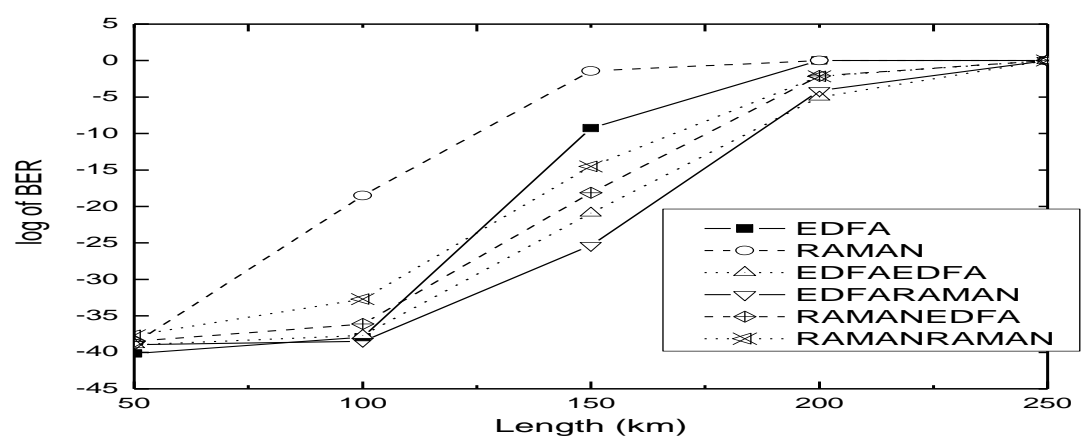

Fig. 3. Diagram Showing log of BER at Different Fiber Length

The variation in log of BER for other is -40.14 to zero for EDFA, -38.82 to zero for RAMAN, -38.51 to zero for RAMAN-EDFA, -37.67 to zero for RAMAN-RAMAN.

The Fig.4 shows the graphical representation of Eye height as a function of distance in the presence of nonlinearities. The maximum eye height is provided by the EDFA-EDFA amplifier for all distance range. RAMAN-EDFA hybrid amplifier also provides better result for the eye height. It is also observed that at $150 \mathrm{~km}$ only EDFA-EDFA has maximum value $\left(5.51 * 10^{-2}\right)$.

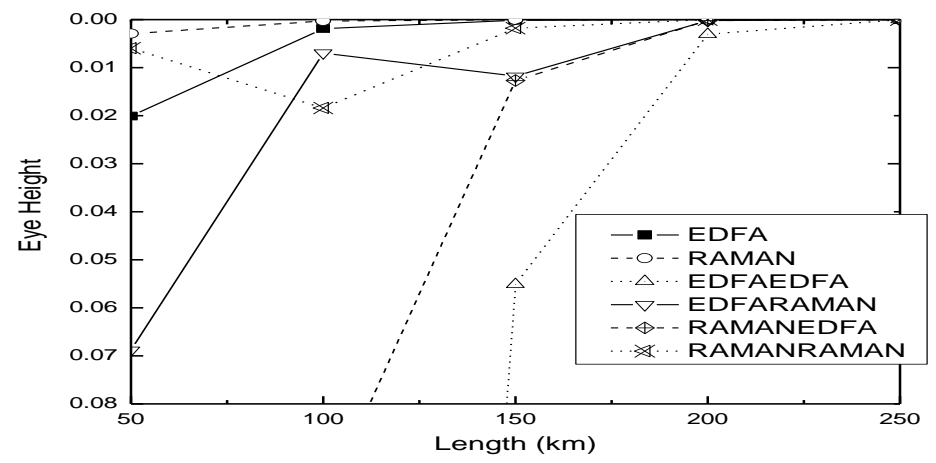

Fig. 4. Diagram Showing Eye Height at Different Fiber Length 
The variation in eye height for other at $150 \mathrm{~km}$ is $\left(1.31952 * 10^{-4}\right)$ for EDFA, $\left(3.42^{*} 10^{-5}\right)$ for RAMAN, $\left(1.17^{*} 10^{-}\right.$

${ }^{2}$ ) for EDFA-RAMAN, $\left(1.26 * 10^{-2}\right)$ for RAMAN-EDFA, $\left(1.79 * 10^{-3}\right)$ for RAMAN-RAMAN.

The Fig. 5 shows the graphical representation of Q-factor vs. Length for 160 channels in the presence of nonlinearities. The Q factor for EDFA-RAMAN amplifier at $100 \mathrm{~km}$ is highest as compare to other which is 11.85 and at $150 \mathrm{~km} 9.38$ for EDFA-EDFA respectively.

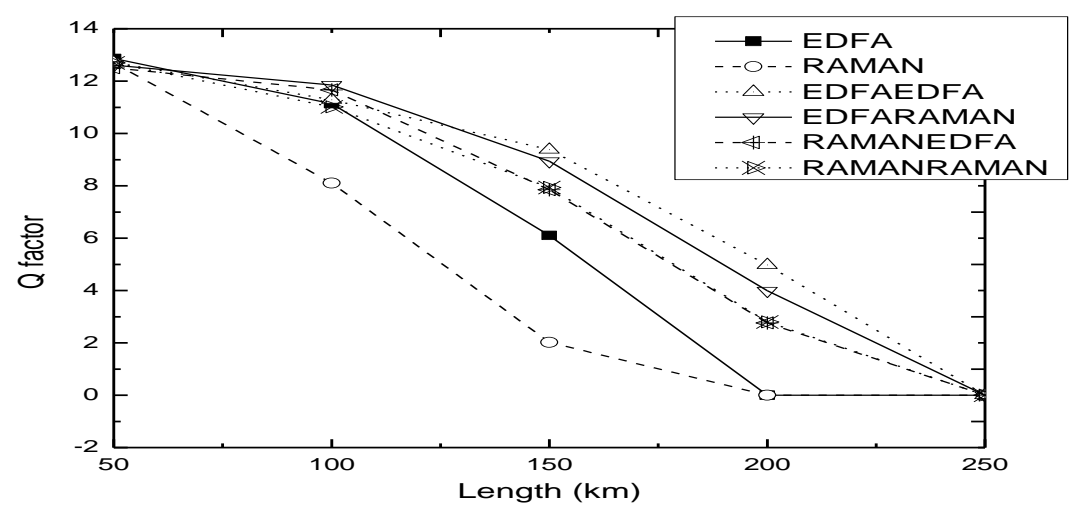

Fig. 5. Diagram Showing Q Factor at Different Fiber Length

The Q-factor for all the configurations is 12.86 to 0 for EDFA, 12.69 to 0 for RAMAN, 12.71 to 4.97 for EDFA-EDFA, 12.60 to 3.99 for EDFA-RAMAN, 12.50 to 2.75 for RAMAN-EDFA,12.69 to 2.79 for RAMAN-RAMAN for 50 to $200 \mathrm{~km}$ range.

\section{Conclusion}

The single and hybrid optical amplifiers for $160 \times 10 \mathrm{Gbps}$ at $25 \mathrm{GHz}$ frequency spacing design models were successfully designed and implemented into Optisystem.v7.0. The main motivation of this work is to optimize the optical amplifiers for different transmission distance and study the nonlinearities effect. The performance of single and hybrid optical amplifiers was evaluated using the output power, BER, eye height and $\mathrm{Q}$ factor. The simulation results show that EDFA-EDFA performed better than other optical amplifiers at 150 $\mathrm{km}$ distance.

By the proposed scheme EDFA-EDFA provide high power (36.55 to $-3.45 \mathrm{dBm})$, least BER (-38.96 to 0 ), large $Q$ factor (12.71 to 0 ) and good eye diagram for different transmission distance ranging from 50 to 250 $\mathrm{km}$. But it is useful up to $150 \mathrm{~km}$ above $150 \mathrm{~km}$ distance; there is more distortion in the received signal. The output power, Q factor and eye height are decreasing above this. Also, there is an increment in BER after 150 $\mathrm{km}$. So, this proposed model for EDFA-EDFA is best suited for $150 \mathrm{~km}$ distance.

\section{References}

[1] H. Masuda, S. Kawai and K. Aida, "Ultra-wideband hybrid amplifier comprising distributed Raman amplifier and erbium doped fiber amplifier", IEEE Electronic Letters, Volume 34, Number 13, 1998, pp. 1342-1344.

[2] Hari Bhagwan, Tarun Gulati and Bharat Rawat, "Evaluation of optical amplifiers", International Journal of Engineering Research and Applications (IJERA) ISSN: 2248-9622, Volume 2, Issue 1, Jan-Feb 2012, pp. 663-667.

[3] M. M. J. Martini, C. E. S. Castellani, M. J. Pontes, M. R. N. Ribeiro and H. J. Kalinowski, "Multi-pump optimization for Raman+EDFA hybrid amplifiers under pump residual recycling", IEEE MTT-S International Microwave \& Optoelectronics Conference, 2009, pp. 117-121.

[4] J. M. P. Delavaux, C. F. Flores, R. E. Tench, T. C. Pleiss, T. W. Cline, D. J. Digiovanni, J. Federici, C. R. Giles, H. Presby, J. S. Major and W. J. Gignac, "Hybrid Er-doped fiber amplifiers at 980-1480 nm for long distance optical communication", IEEE Electronics Letters", Volume 28, Number 17, 1992, pp. 1642-1643.

[5] J. H. Lee, Y. M. Chang, Y. G. Han, H. Chung, S. H. Kim and S. B. Lee, "Experimental performance comparison for a variety of single pump, highly efficient, dispersion compensating Raman/EDFA hybrid amplifiers", Optical Fiber Communication Conference, Volume 4,6-11 March 2005.

[6] X. S. Cheng, R. Parvizi, H. Ahmad and S. W. Harun "Wide-band Bismuth-based Erbium-doped fiber amplifier with a flat-gain characteristic", IEEE Photonics Journal, Volume 1, Number 5, November 2009, pp. 259-264.

[7] Hiroji Masuda and Shingo Kawai "Wide-band and gain-flattened hybrid fiber amplifier consisting of an EDFA and a multi wavelength pumped Raman amplifier”, IEEE Photonics Technology Letters, Volume 11, Number 6, June, 1999 , pp. $647-649$.

[8] Simranjit Singhand R. S. Kaler, "Flat-gain L-band Raman-EDFA hybrid optical amplifier for dense wavelength division multiplexed system”,IEEE Photonics Technology Letters, Volume25, Number 3, February 1, 2013, pp. 250-252. 


\section{BIOGRAPHIES}

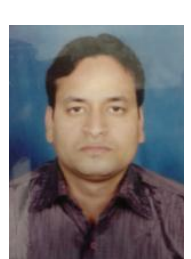

Anil Agarwal was born in Jaipur in Rajasthan State of India on February 11, 1986. He studied at Jaipur Engineering College \&Research Centre Jaipur and received the Electronics \& Communication Engineering degree from Rajasthan University Jaipur, Rajasthan, India in 2007. He is currently Pursuing M.Tech (Communication \& Signal Processing) from Jaipur National University Jaipur, India.

From 2007 to 2008, he was Project Engineer in Reliance Communication Jaipur, Rajasthan. After from 2008 to 2011 he was Assistant Professor with the RCERT, Jaipur. He has been Assistant Professor with Jaipur National University, Jaipur, India since 2011. His special fields of interest are Optical Fiber Communication Systems and Microprocessors.

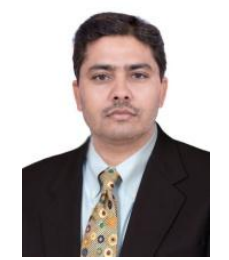

Dr. Sudhir Kumar Sharma is Professor \& Head at the Department of Electronics \& Communication Engineering, School of Engineering and Technology, Jaipur National University, Jaipur, Rajasthan, India. Professor Sharma received his Ph.D. in Electronics from Delhi University in 2000. Professor Sharma has an extensive teaching experience of 17 years. He has been keenly carrying out research activities since last 19 years prominently in the field of Optical Communication. He has taught various engineering courses at graduate as well as at post-graduate level in India and Overseas. His area of research includes Optical Communication, Nonlinear Optics and Optical Sensors. He has authored and coauthored over 60 National and International publications along with one book in Opto-Electronics titled "Introduction to Opto-Electronics and OpticalCommunication", Printice Hall, Malaysia. He has also served on the editorial board of six international journals. He has been part of many National and International conferences worldwide. Professor Sharma continues to serve on many academic, professional and governmental advisory committees. He has organized numerous National and International Workshop, Seminar and Conferences. He has visited Spain, Holland, Malaysia, Ethiopia, Sudan and Syria for academic and research related assignments. He has awarded Senior Research Fellowship by CSIR, New Delhi in 1997. He has also been honored with the prestigious Shiksha Rattan Puruskar by International Friendship Society, New Delhi for academic works in Feb. 2013. As many as 15 M.Tech students have completed their thesis under his guidance and 5 are ongoing at present. He has supervised $3 \mathrm{PhD}$ students successfully and $7 \mathrm{PhD}$ students are currently pursuing their research under guidance. 